\title{
A Generalized Cognitive Hierarchy Model of Games
}

\author{
Juin-Kuan Chong, Teck-Hua Ho, and Colin Camerer*
}

June 4,2014

Subjects in simple games often exhibit nonequilibrium behaviors. Cognitive Hierarchy $(\mathrm{CH})$ and Level $k$ (LK) are two prevailing structural models that can predict these behaviors well but they make quite different assumptions on players' beliefs of their opponents' actions. This paper develops a generalization of $\mathrm{CH}$ and shows that $\mathrm{CH}$ and a variant of $\mathrm{LK}$ belong to the same family. Under generalized $\mathrm{CH}(\mathrm{GCH})$, level $k$ players best respond to level 0 to level $k-1$ but the perceived proportion of each lower level is obtained by weighting its actual frequency by a parameter $\alpha$, reflecting stereotype bias well documented in social psychology literature. When $\alpha=1, \mathrm{GCH}$ reduces to $\mathrm{CH}$; and when $\alpha=\infty$, it becomes Level $m$ (LM) in which level $k$ best responds to only the modal level below $k$ (and the modal level may be $k-1$ ). GCH also fixes prior ad-hoc assumptions about level 0 by developing a plausible model for it. GCH posits that non-strategic level 0 players are more likely to choose strategies that will never yield the minimum payoff in all possible outcome scenarios. This minimum-aversion tendency captures level 0's avoidance for dominated strategies and compromise effects welldocumented in the individual choice literature. Using fifty-five 2-player $m \times n$ games from four distinct datasets, we show that $\mathrm{GCH}$ describes and predicts behaviors better than $\mathrm{CH}$ and LK. Structural estimation results show that higher level players exhibit stereotype bias and level 0 players exhibit minimum aversion. Finally, we apply GCH to two new games and find that $\mathrm{GCH}$ is able to overcome CH's inadequacies and predict behavior remarkably well.

Keywords: Cognitive Hierarchy, Level $k$ Model, Level $m$ Model, Generalized Cognitive Hierarchy, Nonequilibrium Structural Models, Behavioral Game Theory

${ }^{*}$ Chong: National University of Singapore. Ho: University of California, Berkeley and National University of Singapore. Camerer: Caltech. Direct correspondence to Juin-Kuan Chong: bizcjk@nus.edu.sg. We thank Vince Crawford for his encouragement and Sada Reza for his research assistance. 


\section{Introduction}

Subjects frequently do not play equilibrium in simple one-shot games. Sometimes these nonequilibrium behaviors persist even when subjects engage in repeated plays and when they are motivated by substantial financial incentives. Both nonequlibrium phenomena pose serious challenges for standard equilibrium models. Recent research has developed structural nonequilibrium models as alternatives and these models have shown promise in explaining and predicting these out-of-equilibrium behaviors in a wide variety of games and markets (see Crawford (2013) for a comprehensive review).

Standard equilibrium models make three fundamental assumptions to generate their prediction: 1) players form subjective beliefs about what their opponents will do, 2) players choose actions to maximize their expected payoffs conditional on their belief (i.e., they are subjective utility maximizers), and 3) players' subjective beliefs are always correct (i.e., their beliefs always match their opponents' actual actions). The third assumption is particularly strong because it uses opponents' actual actions to pin down players' beliefs. Since players' beliefs are always correct under standard equilibrium models, no players will ever be surprised by their opponents' actions. Nonequilibrium structural models, on the other hand, do not require players' subjective beliefs to be always correct. As a result, players can be surprised by their opponents' actions in actual game plays (as we observe in many real-world situations).

To generate their behavioral prediction, existing nonequilibrium models posit that players are heterogenous and have different levels of thinking ability. The proportion of player with thinking level $k(k=0,1, \ldots, \infty)$ is denoted by $f(k)$. Clearly, $\sum_{k=0}^{\infty} f(k)=1$. These models starts with an explicit assumption on how non-strategic level 0 players behave. Higher level players' behaviors are then determined iteratively by assuming they best-respond to lower

level players. As a consequence, the aggregate behavioral prediction is simply an average of each level's behavior weighted by its actual proportion.

Cognitive Hierarchy (CH) (Camerer, Ho and Chong, 2004) and Level $k$ (LK) (Nagel, 1995; Stahl and Wilson, 1994, 1995; Costa-Gomes, Crawford and Broseta, 2001; Costa-Gomes and Crawford, 2006) have been successful in explaining nonequilibrium behaviors, however, they 
make quite different assumptions on how higher level players best respond to lower level players. Both models start with a specification of level 0 rule. While LK assumes that level $k$ best-responds to only level $k-1, \mathrm{CH}$ assumes that level $k$ best respond to all lower level (i.e., $0,1, \ldots, k-2, k-1$ ) with the perceived proportion of each lower level equals to its normalized true proportion (i.e., perceived proportion of level $h=\frac{f(h)}{\sum_{h^{\prime}=0}^{k-1} f\left(h^{\prime}\right)}$, for all $h<k$ ). This paper develops a generalization of $\mathrm{CH}$ and shows that it nests a variant of LK, which we call Level $m$ (LM) model. Like LK, LM assumes each level of player best-responds to only one lower level player. Unlike LK, however, LM assumes level $k$ best-responds to the most frequently occurring lower level (i.e. the modal level below $k$ ), reflecting stereotype bias. If the modal level happens to be $k-1$, LK and LM make the same prediction for level $k$ behavior.

Generalized Cognitive Hierarchy (GCH) assumes that level $k$ best-responds to all lower levels but the perceived proportion of each lower level is obtained by weighting its actual frequency by a parameter $\alpha$. Specifically, level $k$ 's belief of the proportion of level $h(<k)$, the perceived proportion of level $h$, is equal to $\frac{f(h)^{\alpha}}{\sum_{h^{\prime}=0}^{k-1} f\left(h^{\prime}\right)^{\alpha}}$. As a result, GCH reduces to $\mathrm{CH}$ when $\alpha=1$ and LM when $\alpha=\infty$. Note that when $\alpha=\infty$, perceived proportion of level $h=1$ if $h=\operatorname{argmax}_{h^{\prime}<k} f\left(h^{\prime}\right)$ and 0 otherwise. We shall show below that LM avoids an undesirable property of LK, which predicts oscillating player behavior in some games including market entry.

The behavioral prediction of non-equilibrium structural models depends critically on level 0's behavioral rule because higher level rules are defined iteratively based on lower level ones. Despite its importance, there is a lack of a plausible model for level 0's rule that can be applied uniformly to all games. Existing research uses two different models of level 0's rule. In one model, level 0 is assumed to randomize her choice uniformly across all possible actions (e.g., Camerer et al, 2004). While the random choice rule is general, it appears too naive as a plausible rule of behavior for actual players in practice. In another model, saliency is used to derive level 0's rule from the game structure (e.g., Crawford and Iriberri, 2007). This saliency-based model, while plausible, can sometimes appear ad-hoc. This is so because saliency is not precisely defined and different people may invoke a different saliency principle.

In this paper, we develop a model of level 0 that aims to address the inadequacies of existing 
models of level 0 . Our model hypothesizes that level 0 players use minimum avoidance as the saliency principle in choosing their strategy. Specifically, they prefer strategies that will not yield the minimum payoff in all possible outcome scenarios. Let's illustrate the minimum avoidance principle with a $n \times m$ game matrix. We focus on the row player $i$ who has $n$ strategies. Strategy $j$ belongs to the preferred set $S_{i}$ if strategy $j$ never yields the minimum payoff in any of the $m$ possible opponent's strategies. Our model posits that level 0 players choose strategies in set $S_{i} \beta$ times more likely than those outside $S_{i}$. As a consequence, level 0 players choose each of the strategies in $S_{i}$ with probability $\frac{\beta}{n-\left|S_{i}\right|+\left|S_{i}\right| \cdot \beta}$ and each of the strategies not in $S_{i}$ with probability $\frac{1}{n-\left|S_{i}\right|+\left|S_{i}\right| \cdot \beta}$, where $\left|S_{i}\right|$ is the cardinality of the set $S_{i}$. Below, we show that this simple model of level 0's rule exhibits dominance and compromise effects, two behavioral phenomena well-documented in the individual choice literature. In addition, it predicts that level 0 is less likely to choose dominated strategies, which fixes a weakness of the random choice model. Note that when $\beta=1$, the model reduces to random choice rule. When $\beta=\infty$, level 0 never chooses strategies outside the preferred set $S_{i}$.

GCH has 2 more parameters (i.e., $\alpha$ and $\beta$ ) than the $\mathrm{CH}$ model. The $\alpha$ parameter allows us to investigate various models of opponents in a common framework. It allows us to establish an explicit link between $\mathrm{CH}$ and a variant of LK (i.e., LM). The $\beta$ parameter uses minimum avoidance as the saliency principle and allows us to model random choice and saliency as two extreme special cases of a well-defined model of level 0.

We fit GCH to $55 n \times m$ games (with $n, m=2,3,4,5,6)$ from 4 distinct datasets. We show that our generalized model describes and predict behaviors better than $\mathrm{CH}$ and LK models. Both $\alpha$ and $\beta$ are important and add to the overall fit. The parameter $\alpha$ is always greater 1 , suggesting that higher level players exhibit stereotype bias. The parameter $\beta$ is always greater than 1 , suggesting that level 0 players exhibit minimum aversion. We also estimate nested cases of GCH and show that they are rejected in favor of the general model.

To check for robustness, we apply GCH to predict behavior in two new games. In all games, $\mathrm{CH}$ did not predict the observed behaviors well. In the first game, Carrillo and Palfrey (2009) find that people tend to fight in a fight-or-compromise game less frequently than Nash equilibrium would predict. We show that GCH is able to overcome CH's shortcomings and predict observed behaviors remarkably well. In the second game, Amaldoss, Bettman 
and Payne (2008) add an irrelevant (i.e., dominated) alternative to a coordination game and show that subjects are better able to coordinate their behavior. GCH is able to capture the better coordination.

The rest of the paper is organized as follows. Section 2 describes the model. It shows how the GCH model nests CH and LM as special cases. Section 3 compares LM and LK models in several games (including the entry game) and describes their similarities and differences. It shows that LM does not exhibit oscillating behavior. Section 4 provides MLE estimates of GCH, CH, LM, LK and a 2-parameter LK models. Section 5 describes two applications of best fitted GCH to 2 new games. Section 6 concludes and suggests future research directions.

\section{Model}

\section{$2.1 \quad$ Notations}

We focus the GCH model on 2-player matrix games. Beginning with notation, we index players by $i$ and denote player $i$ 's $j$ th strategy by $s_{i}^{j}$. Player $i$ chooses from $m_{i}$ possible strategies. Player $i$ 's opponent is denoted by $-i$ and her strategy profile by $s_{-i}^{j^{\prime}}$, and there are $m_{-i}$ such strategies. Player $i$ 's payoff is denoted by $\pi_{i}\left(s_{i}^{j}, s_{-i}^{j^{\prime}}\right)$. Players are heterogenous and choose a rule from a rule hierarchy. We index rule levels in the rule hierarchy by $k$ $(k=0,1,2, \ldots, k-1, k, \ldots, \infty)$ and denote the proportion of level $k$ players by $f(k)$. Clearly $\sum_{k=0}^{\infty} f(k)=1$. In the empirical estimation, we assume $f($.$) follows a 1-parameter Poisson$ distribution with mean and variance $\tau$. Formally, $f(k)=\frac{\tau^{k} \cdot e^{-k \cdot \tau}}{k !}$.

\subsection{Model of Opponent}

Denote the probability that a player $i$ with rule level $k$ choosing strategy $s_{i}^{j}$ by $P_{k}\left(s_{i}^{j}\right)$. Like $\mathrm{CH}, \mathrm{GCH}$ posits that level $k$ best responds to level 0 to level $k-1$ and chooses the strategy that maximizes her expected payoff. The expected payoff of choosing $s_{i}^{j}$ is computed on the basis of level $k$ 's belief on what the lower levels will do. Denote the expected payoff of strategy $s_{i}^{j}$ for level $k$ as $E_{k}\left(s_{i}^{j}\right)$. The expected payoff is computed as follows: 


$$
E_{k}\left(s_{i}^{j}\right)=\sum_{j^{\prime}=1}^{m_{-i}} \pi_{i}\left(s_{i}^{j}, s_{-i}^{j^{\prime}}\right)\left\{\sum_{h=0}^{k-1} g_{k}(h) \cdot P_{h}\left(s_{-i}^{j^{\prime}}\right)\right\}
$$

where $g_{k}(h)$ is level $k$ 's belief on the proportion of level $h, h<k$.

A level $k$ player believes that the relative proportion of lower level $h$ is:

$$
g_{k}(h)=\frac{f(h)^{\alpha}}{\sum_{i=0}^{k-1} f(i)^{\alpha}}, \quad \forall h<k .
$$

The parameter $\alpha \geq 1$ captures the tendency of players exhibiting stereotype bias. When $\alpha=1$, level $k$ 's belief of lower level equals to its normalized true proportion and hence correctly reflecting the relative proportions of the lower levels. When $\alpha>1$, player $k$ 's belief of lower levels is concentrated on the more frequently occurring levels. This bias is the stereotype bias. Stereotype bias is well documented in social psychology literature. Stereotype bias is a simplification of beliefs (where heterogeneity of outgroup members is reduced) when people are faced with heavy cognitive load or when their cognitive resources are depleted. This simplification of belief is done to achieve efficiency in information processing and decision making.

Note that when $\alpha=1$, GCH reduces to $\mathrm{CH}$. When $\alpha=\infty$, level $k$ exhibits extreme stereotype bias and believes that her opponent is only of the most frequently occurring lower rule. We call this special case the Level $m$ (LM), in contrast to LK, which places the entire weight on level $k-1$. Both LK and LM assume that level $k$ player believes that his opponent is of a singular type; the type is level $k-1$ in LK and the modal lower rule in LM. If the modal rule is always level $k-1, \forall k$, then the two models are identical. For well-behaved single-modal distributions (e.g., Poisson), the two models generate an identical prediction for level $k$ up to the mode +1 and a different prediction for level $k$ above mode +1 . 


\subsection{Model of Level 0}

As discussed below, there are two existing models of level 0 rule. One model assumes that level 0 uniformly randomizes among all possible strategies. This model has the advantage that it is well-defined and general. However, it does not appear plausible for describing behaviors of sufficiently motivated subjects. The second model assumes that Level 0 chooses a salient action in the feasible strategy space. This model receives the criticism of being ad-hoc because it is often unclear ex ante which action is salient in some games. GCH addresses the inadequacies of these models by proposing a model of level 0 that is both behaviorally plausible and empirically general.

GCH assumes that level 0 players are averse to receiving minimum payoffs. Specifically, the model assumes that level 0 is more likely to choose from a set of strategies that will never yield the minimum payoff in any outcome scenario. That is, a strategy in this set will always provide a strictly higher payoff than at least one other strategy in any outcome scenario. These strategies are never the worst.

For player $i$, we define $i$ 's "never worst set" $S_{i}$ as the the set of strategies that will never yield the worst payoff in any of the possible strategies of opponent $-i$. Specifically, any strategy $j$ in set $S_{i}$ will yield a strictly higher payoff than at least one other strategy in every possible strategy of $-i$. Formally, if $i$ 's strategies are indexed by $j$ as well as $j^{\prime}$ and $-i$ 's strategies are indexed by $j^{\prime \prime}$, then $S_{i}=\left\{j: \pi_{i}\left(s_{i}^{j}, s_{-i}^{j^{\prime \prime}}\right)>\min _{j^{\prime}}\left\{\pi_{i}\left(s_{i}^{j^{\prime}}, s_{-i}^{j^{\prime \prime}}\right), \quad \forall j^{\prime \prime}\right\}\right.$. GCH posits that level 0 player $i$ choose strategies in $S_{i} \beta$ times more likely those not in $S_{i}$. Hence, the choice probability of level 0 for each strategy $s_{i}^{j}$ is as follows:

$$
P_{0}\left(s_{i}^{j}\right)=\left\{\begin{array}{lcc}
\frac{\beta}{m_{i}-\left|S_{i}\right|+\left|S_{i}\right| \cdot \beta} & \text { if } & j \in S_{i} \\
\frac{1}{m_{i}-\left|S_{i}\right|+\left|S_{i}\right| \cdot \beta} & \text { otherwise. }
\end{array}\right.
$$

where $\left|S_{i}\right|$ is the cardinality of $S_{i}$. Note that $S_{i}$ can be a null set and in this case our model reduces to the uniform randomization rule with level 0 players choose each strategy with an equal probability of $\frac{1}{m_{i}}$.

The proposed model of non-strategic level 0 captures two well-established empirical regularities documented in the individual choice literature: 1) dominant strategies are chosen more 
frequently than dominated strategies, and 2) compromise effects. In addition, the model also captures the asymmetric dominance phenomena when the dominant strategy does not have a minimum. The following three examples illustrate these phenomenas.

\section{Existence of Dominant Strategies}

When player $i$ has 2 strategies and one of them is strictly dominant, level 0 player chooses this strategy with probability of $\frac{\beta}{1+\beta}$. Table 1 shows one such game (Game 2 from Cooper and Van Huyck, 2003). The dominant strategy is labeled T, which pays 0.4 and 0.6 when the opponent chooses $\mathrm{L}$ and $\mathrm{R}$ respectively. It is more than what strategy $\mathrm{B}$ will pay in both scenario which is 0.2 . GCH allows us to investigate whether by allowing level 0 to choose $\mathrm{T} \beta$ times more likely than $\mathrm{B}$ would help to describe and predict behaviors better in this kind of games. There are 10 games that have a dominant strategy for at least one of the two players in the 55 games we studied.

Table 1: Game 2 from Cooper and Van Huyck [2003]

\begin{tabular}{l|cc|c}
\hline \hline & $\mathrm{L}$ & $\mathrm{R}$ & data \\
\hline $\mathrm{T}$ & $.4, .5$ & $.6, .3$ & .909 \\
$\mathrm{~B}$ & $\mathbf{. 2 , . 1}$ & $\mathbf{. 2}, \mathbf{. 1}$ & .091 \\
\hline data & .738 & .262 & \\
\hline
\end{tabular}

\section{Compromise Effects}

Decision makers have a tendency to select a compromise alternative in individual choice. Specifically, in a choice among three alternatives with 2 attributes, they often prefer the alternative that yields intermediate payoffs in both attributes to alternatives that yield high payoff in one attribute and low payoff in the other attribute. This behavioral phenomenon of minimum aversion is called compromise effect. If players exhibit compromise effect in games, they may choose a strategy that never yields the 
minimum payoff. We use Game 10 from Camerer et al (2004) to illustrate this behavioral tendency. Strategy $\mathrm{M}$ of row player is a compromise alternative because it gives intermediate payoffs of -10 and 0 when the column player chooses $\mathrm{L}$ and $\mathrm{R}$ respectively. It also gives the maximum payoff of 30 when column player chooses C. Note that strategy $\mathrm{M}$ never receives the worst payoff in all possible outcome scenarios. GCH predicts that level 0 chooses $\mathrm{M}$ with probability $\frac{\beta}{2+\beta}$ and $\mathrm{T}$ or $\mathrm{B}$ with probability $\frac{1}{2+\beta}$. Interestingly, subjects in this game play Strategy M with a frequency of $96 \%$. In the 55 games we analyze, there are 10 games with such payoff structure.

Table 2: Game 10 from Camerer et al [2004]

\begin{tabular}{l|ccc|c} 
& $\mathrm{L}$ & $\mathrm{C}$ & $\mathrm{R}$ & data \\
\hline $\mathrm{T}$ & $\mathbf{- 2 0 , 2 0}$ & $30, \mathbf{- 3 0}$ & $\mathbf{- 3 0 , 3 0}$ & .00 \\
$\mathrm{M}$ & $-10,10$ & 30,30 & $0, \mathbf{0}$ & .96 \\
$\mathrm{~B}$ & 0,0 & $\mathbf{- 1 0 , 1 0}$ & $\mathbf{1 0 , - \mathbf { 1 0 }}$ & .04 \\
\hline data & .29 & .58 & .13 & \\
\hline
\end{tabular}

\section{Asymmetric Dominance}

Sometimes there is no dominant strategy in a $2 x 2$ game. One way to make a strategy more attractive is by adding a dominated strategy to the feasible strategy space. Equilibrium models predict that players will not behave differently with the addition of irrelevant alternative. However experimental literature suggests that players often choose the strategy that dominates the added (and dominated) strategy more frequently. We use Game 6 from Costa Gomes et al (2001) to illustrate this phenomenon. This game is a 3x2 matrix game with payoff matrix as shown in Table 3. Note that strategy $\mathrm{M}$ is a dominated strategy (it is dominated by $\mathrm{B}$ ). If players exhibit asymmetric dominance, they will be more likely to choose strategy B. Indeed, B is played $75 \%$ of the time by the row players even though this game has a unique pure strategy Nash 
equilibrium at $(\mathrm{T}, \mathrm{L})$. GCH can capture this phenomenon since its level 0 chooses B with probability $\frac{\beta}{2+\beta}$ and strategy $\mathrm{T}$ and $\mathrm{M}$ with probability $\frac{1}{2+\beta}$. There were 7 games with asymmetric dominance payoff structure for at least of one of the two players in the 55 games we studied.

Table 3: Game 6 from Costa Gomes et al [2001]

\begin{tabular}{l|cc|c} 
& $\mathrm{L}$ & $\mathrm{R}$ & data \\
\hline $\mathrm{T}$ & 74,62 & $\mathbf{4 3 , 4 0}$ & .14 \\
$\mathrm{M}$ & $\mathbf{2 5 , 1 2}$ & 76,93 & .11 \\
$\mathrm{~B}$ & 59,37 & $94, \mathbf{1 6}$ & .75 \\
\hline data & .70 & .30 & \\
\hline \\
Note: Payoffs in bold indicate the minimum payoff for \\
the corresponding columns or rows. The never worst set \\
for the row player consists of strategy B which domi- \\
nates strategy M. The never worst set for column player \\
is empty.
\end{tabular}

In sections 4 and 5, we provide precise measure of statistical significance for the GCH model. Before doing so, we would like to elaborate on one important feature of the GCH model. We show how a special case of the GCH model is linked to the Level $k$ model in the next section.

\section{Level $m$ versus Level $k$}

Our Level $m$ (LM) and Level $k$ (LK) models share a common feature: both have players higher than level 0 best-responding to a single lower level. As a consequence, they are more tractable analytically. However, they differ in which specific lower level the higher level best-responds to. The LM model assumes level $k$ best responds to the mode of the lower levels while LK model assumes level $k$ best responds to level $k-1$. When $k$ is small, the two models frequently make the same prediction. Indeed, if the distribution of thinking level is unimodal, the two models predict the same lower level for $k$ up to one level higher than 
the mode. As a consequence, the two models make different predictions only among players whose level $k>$ mode +1 .

To understand the extent of difference between the two models under different mean thinking step $\tau$, we compute the proportion of predicted behaviors which are different between the two models. Figure 1 below shows four lines. The top most line represents the theoretical upper bound for the proportion of difference. It is computed from the proportion of all level $k$ players in the LM model who do not best respond to level $k-1$. $^{1}$ The figure is generated for $\tau$ in the range of 0 and 4 . We also generate empirical proportion of all level $k$ players in the LM model who do not pick the same strategy as the level $k$ in the LK model for each of the 55 games we considered. These empirical proportions should be lower than the upper bound since level $k$ who does not best respond to level $k-1$ in LM model may be predicted to best respond with the same strategy as level $k$ in LK model who best responds to level $k-1 .^{2}$ The thick dashed line indicates the maximum proportion from the 55 games. As we can see the maximum proportions practically matches the upper bounds over the entire range of $\tau$. We also report the median and the minimum. The minimum is always zero suggesting that for each $\tau$, there are games in which LM and LK produce the same prediction.

A drawback of LK model is that it may lead to oscillating behavior as the thinking level increases. To see this, let us consider Game 7 of Stahl and Wilson (1995). This game is a 3x3 symmetric matrix game (see Table 4). To determine LK's prediction in this game, let us begin with the choice of level 1 . Believing that her opponent is level 0 who chooses the three strategies equally likely, level 1 best responds by choosing $\mathrm{T}$ as it offers the highest expected payoff of $\frac{1}{3} \cdot 30+\frac{1}{3} \cdot 100+\frac{1}{3} \cdot 50=60$. Level 2 best responds to level 1's choice of $\mathrm{T}$ by choosing $\mathrm{B}$ which gives a payoff of 50 . Level 3 best responds to level 2's choice of B by choosing M yielding a payoff of 90. Level 4 best responds to level 3's choice of M by choosing $\mathrm{T}$ paying 100. Level 5 will best respond to $\mathrm{T}$ with a choice of $\mathrm{B}$, as what a level 2 will do; and the higher levels will cycle through the same choice sequence of T, B and M endlessly. Given the empirical frequency as reported in Table 4, the best fitted $\tau$ for LK model is 0.166.

Does LM model suffer from the same drawback of oscillating behavior? The answer is no.

\footnotetext{
${ }^{1}$ That is, we compute for the LM model, $\sum_{k} f(k) \cdot I\left[g_{k}(k-1) \neq 1\right]$ where $I[\cdot]$ is the indicator function.

${ }^{2}$ The proportion is $\sum_{k} f(k) \cdot I\left[P_{k}^{L M}\left(s_{i}^{j}\right) \neq P_{k}^{L K}\left(s_{i}^{j}\right)\right]$ where $I[\cdot]$ is the indicator function.
} 
FIGURE 1

Proportion of Different Predicted Behaviors between LM and LK Models

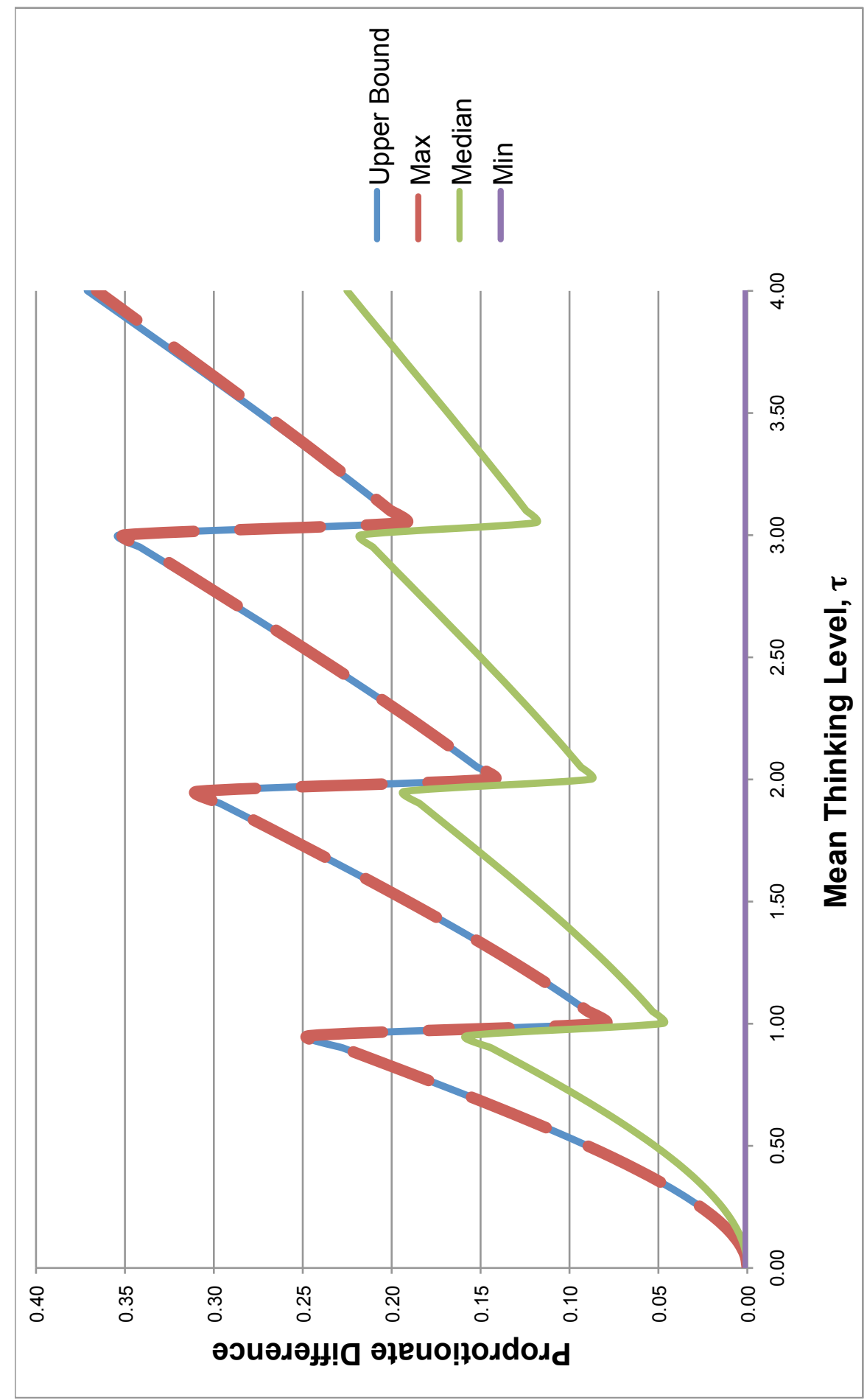


Table 4: Game 7 from Stahl and Wilson [1995]

\begin{tabular}{c|ccc|c}
\hline \hline & $\mathrm{T}$ & $\mathrm{M}$ & $\mathrm{B}$ & data \\
\hline $\mathrm{T}$ & $\mathbf{3 0}$ & 100 & 50 & .44 \\
$\mathrm{M}$ & 40 & $\mathbf{0}$ & 90 & .35 \\
$\mathrm{~B}$ & 50 & 75 & $\mathbf{2 9}$ & .21 \\
\hline \hline
\end{tabular}

Note: Payoffs in bold indicate the minimum payoff for the
corresponding columns or rows. Best response to strategy
$\mathrm{T}$ is strategy $\mathrm{B}$, best response to strategy $\mathrm{B}$ is strategy $\mathrm{M}$
and best response to strategy $\mathrm{M}$ is strategy $\mathrm{T}$, making it
a full cycle.

Given the empirical data, the best fitted $\tau$ for LM model is 0.178. Under LM, Level 1's best response is T, which follows from the same calculation as the LK model. Level 2 best responds to the most frequently occurring lower type which in this case is level 0 (since $\tau=0.178$ ). Hence, the choice of level 2 is $\mathrm{T}$, same as level 1 . The modal type is level 0 for all higher type; hence, all higher levels also choose $\mathrm{T}$ as the best response. As a consequence, there is no oscillating behavior among higher level thinkers. ${ }^{3}$

This oscillating behavior of the LK model can also occur in games with $N>2$ players. We illustrate this in a market entry game. In this game, $N$ players independently and simultaneously decide whether or not to enter a market with demand $d$ (where $d<N$ ). If the number of entry is greater than $d$, all entrants earn nothing; otherwise they receive a payoff of 1. Players who stay out receive a payoff of 0.5 . Hence, the best response function for a player depends on the number of other players who choose to enter.

Let us derive the best response function of a level $k$ player. Denote the best response function (or the entry function) of level $k$ with demand $d$ by $e(k, d)$. In addition, let $E_{k}(k-1, d)$ be level $k$ player's assessment of the total number of entries by her competitors who are level $k-1$ or lower. Hence the entry function of level $k$ player can be defined as follows:

\footnotetext{
${ }^{3}$ In this game, LM fits slightly better than LK. There is a $0.32 \%$ improvement in log-likelihood (note that both models have one parameter).
} 


$$
e(k, d)=\left\{\begin{array}{lll}
0 & \text { if } & E_{k}(k-1, d)>d \\
1 & \text { if } & E_{k}(k-1, d)<d
\end{array}\right.
$$

Using the above equation, the entry function can be recursively derived starting with $e(0, d)$. In the case of the LK model, a level $k$ player believes that she is facing only level $k-1$ players, hence $E_{k}(k-1, d)=e(k-1, d)$. Using the entry function in equation (4), level $k$ will enter if $k-1$ stays out and vice versa. Therefore, we will see alternate entry and no entry decisions being taken as $k$ goes from level 1 to $\infty$. In the case of the LM model, the entry function depends on the mode of distribution of think level. Assuming a single modal distribution such as the Poisson distribution, a level $k$ player, where $k \leq$ mode, believes she is facing only level $k-1$ players, and hence her entry function is best responding to $E_{k}(k-1, d)=e(k-1, d)$. For $k>$ mode, the level $k$ player believes that she is facing only modal players and best respond to $E_{k}(k-1, d)=e($ mode,$d)$. In other words, these players will all best respond to the modal level by making the same decision; if the modal player enters, all higher level will stay out and vice versa. Therefore, we see that the prediction of the LM model converges at levels higher than the mode of the distribution.

In addition to avoiding oscillating behaviors, an equally important empirical question is whether total entry monotonically increases in demand as observed in data? Put simply, if we fix the number of players $N$ but increases the demand $d$, would we observe a corresponding increase in entry from these $N$ players. We show in Appendix 1 that with the assumption of Poisson distribution, the monotonicity in entry is guaranteed when $\tau \leq 1.256$, similar to the condition derived for the $\mathrm{CH}$ model in Camerer et al (2004).

\section{Estimation and Results}

\subsection{Likelihood Function}

Let's first derive the likelihood function. Recall that GCH has three parameters, namely, $\tau, \alpha, \beta$. Let $\left.P_{k}\left(s_{i}^{j} \mid \tau, \alpha, \beta\right)\right)$ be GCH's predicted probability of level $k$ choosing strategy $s_{i}^{j}$. 
We start with level 0 players. $\left.P_{0}\left(s_{i}^{j} \mid \tau, \alpha, \beta\right)\right)=\frac{\beta}{m_{i}-\left|S_{i}\right|+\left|S_{i}\right| \cdot \beta}$ if $s_{i}^{j}$ is in set $S_{i}$ and $\frac{1}{m_{i}-\left|S_{i}\right|+\left|S_{i}\right| \cdot \beta}$ if $s_{i}^{j}$ is not. For $k \geq 1$, we use equation (1) to compute the expected payoff for each strategy $s_{i}^{j}$. Players are assumed to choose the strategy that yields the highest expected payoff. Specifically, $\left.P_{k}\left(s_{i}^{j} \mid \tau, \alpha, \beta\right)\right)=1$ if $s_{i}^{j}$ gives the highest expected payoff and 0 otherwise. ${ }^{4}$ The predicted choice probability for strategy $s_{i}^{j}$ is simply an aggregation of best responses from all thinking levels weighted by their proportion $f(k \mid \tau)$ :

$$
R\left(s_{i}^{j} \mid \tau, \alpha, \beta\right)=\sum_{k} f(k \mid \tau) \cdot P_{k}\left(s_{i}^{j} \mid \tau, \alpha, \beta\right)
$$

where $R\left(s_{i}^{j} \mid \tau, \alpha, \beta\right)$ is the aggregate choice probability of strategy $s_{i}^{j}$.

We then aggregate over all subjects $i$ and all strategies $j$ to form the log-likelihood function as follows:

$$
L L(\tau, \alpha, \beta)=\sum_{i} \sum_{j} I\left(s_{i}^{j}\right) \cdot \ln R\left(s_{i}^{j}, \tau, \alpha, \beta\right)
$$

where $I\left(s_{i}^{j}\right)$ is the indicator function; $I\left(s_{i}^{j}\right)=1$ if player $i$ chooses strategy $s_{i}^{j}$ and 0 otherwise.

We maximize the above log-likelihood function to find the best values for $\tau, \alpha$ and $\beta$. This log-likelihood function can be highly non-linear. To avoid trapping in local optimum, we perform an extensive grid search over the parametric space with $\tau$ ranges from 0 to $10, \alpha$ ranges from 1 to 20 , and $\beta$ from 1 to 10 . It is computationally very demanding so we run multiple grid searches in parallel. We have also truncated the thinking levels at 20 (because the proportion of think ing levels higher than 20 is usually less than 0.00001 empirically). The grids for all parameters are set at intervals of 0.01 .

We calibrate GCH, its special cases, LK and a 2-parameter LK models on the four datasets of matrix games. These include, 12 games in Stahl and Wilson (1995), 8 games in Cooper and Van Huyck (2003), 13 games in Costa Gomes et al (2001) and 22 games collected by Camerer

\footnotetext{
${ }^{4}$ If more than one strategies give the highest expected payoff, we assume players choose the strategies equally likely.
} 
et al $(2004)^{5}$. General details of the games are reported in Table 5. ${ }^{6}$ The four datasets are all 2-person normal form one-shot matrix games. The number of strategies varies from 2 to 6. The whole collection of 55 games offers not only diversity in number of strategies, but more importantly diversity in distinct characteristics of payoff matrices constructed to test various behavioral hypotheses. Hence, the collection of games provides a rigorous test of the GCH model.

\subsection{Results}

Using the 55 matrix games, we estimated the GCH model, its 2 special cases (namely $\mathrm{CH}$ and LM) and LK and a 2-parameter LK models. The 2-parameter LK model allows for a bimodal distribution of types where $\gamma$ proportion of the population is level 0 and $1-\gamma$ proportion of the population follows the Poisson distribution. When $\gamma=0$, the 2-parameter model reduces to the original LK model. Recall that GCH has three parameters $\tau, \alpha, \beta$. The $\mathrm{CH}$ model has $\alpha=1, \beta=1$. The LM model has $\alpha=\infty, \beta=1$. Left panel of Table 6 reports the fit and parameter estimates of the five models. GCH's improvement in fit over the other four models range from $1.2 \%$ to $4.5 \%$. These improvements are significant for two reasons. First, GCH adds an average of 100 likelihood points over the other 4 models; this difference is large when compared with the differences in log-likelihood among the other 3 models. Second, it is difficult to have substantial improvement over the existing models in games with 2 to 3 strategies; 48 of the 55 games we consider are such games.

The $\tau$ estimates of GCH, CH, LM, and LK are remarkably close and between 1.00 and 1.11. ${ }^{7}$ This suggests that the average step of thinking is about $1 . \alpha$ is reliably estimated to be 2.10, suggesting that subjects exhibit stereotype bias and give disproportionately more weight to more frequently occurring levels. $\beta$ is reliably estimated to be 2.10 , indicating that level 0 players exhibit minimum aversion and choose strategies that never see the mini-

\footnotetext{
${ }^{5}$ The 22 games collected by Camerer, Ho and Chong (2004) replicate games reported in Ochs(1995), Bloomfield (1994), Binmore, Swierzbinski and Proulx (2001), Rapoport and Amaldoss (2000), Tang (2001), Goeree, Holt and Palfrey(2003), Mookerjhee and Sopher (1997), Rapoport and Boebel (1992), Messick (1967), Lieberman (1962) and O’Neil (1987).

${ }^{6}$ The payoff matrices with data are available from the corresponding author.

${ }^{7}$ The $\tau$ estimate for the 2-parameter LK model is 1.79 and the bimodal estimate of level 0 is $\gamma=0.34$. This proportion of level 0 estimated by the 2-parameter LK model is quite close to those estimated by the other models. The range of level 0 for Poisson distribution when $\tau=1.00$ and 1.11 is between 0.33 and 0.37 .
} 
Table 5: Data Descriptions

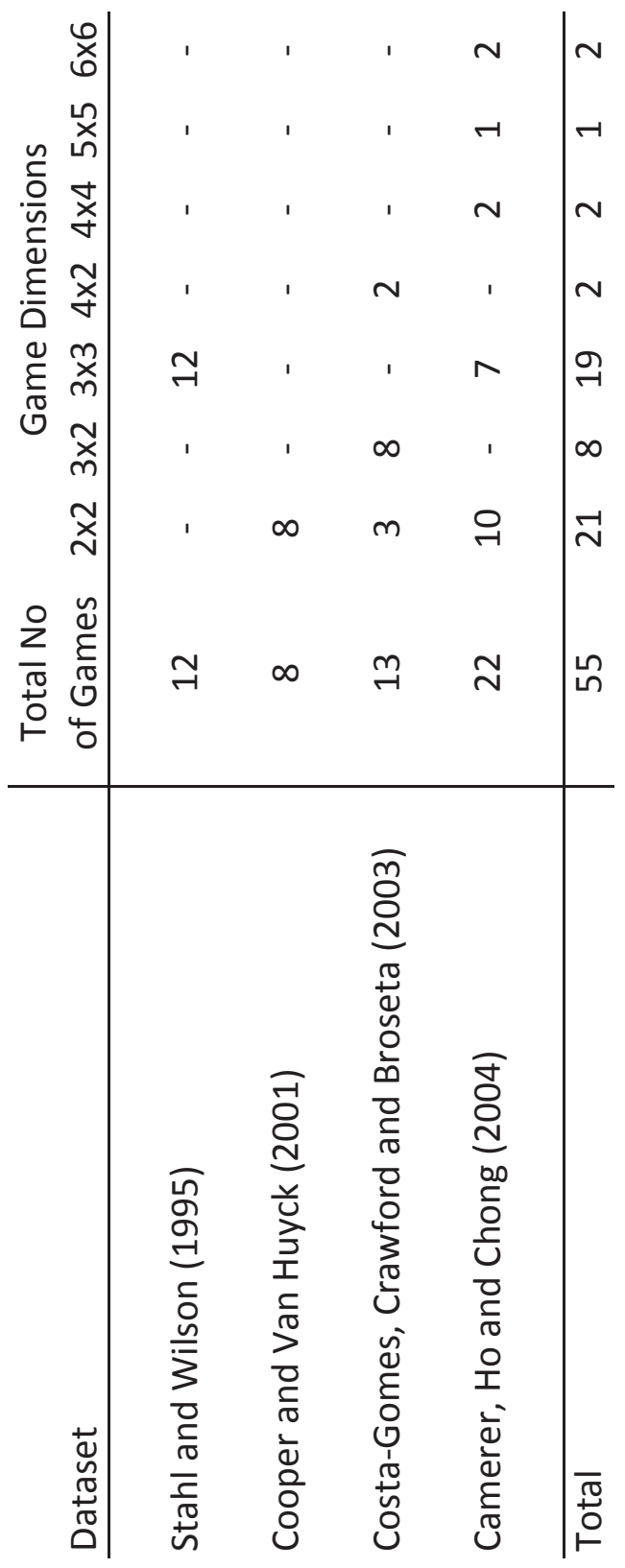


Table 6: Calibration Results for GCH, CH, LM, LK, GCH with $\alpha=1$ and GCH with $\beta=1$

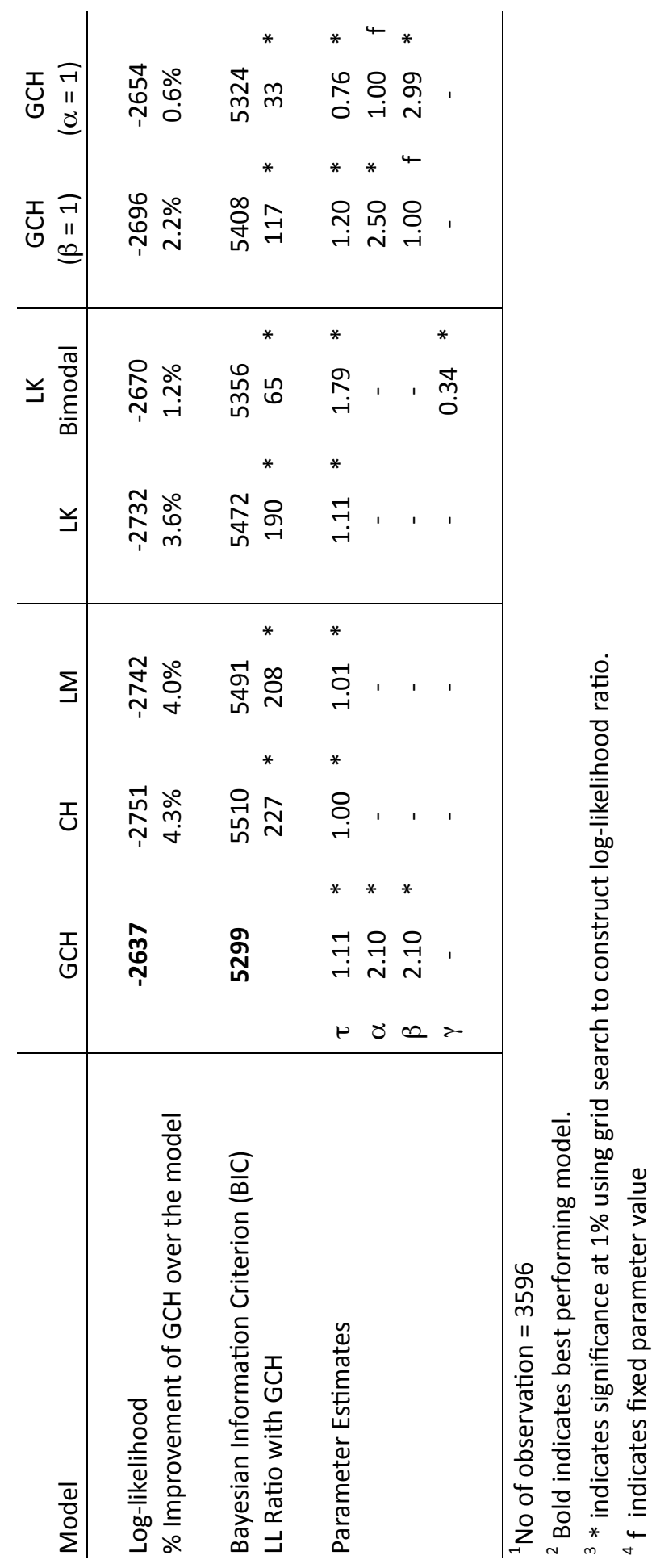


mum payoff twice as likely as those that may see the minimum payoff in at lease one outcome.

To check that the two modeling extensions are significant individually, we also estimate the special cases of $(\alpha, \beta=1)$ (which provides a new model of opponents) and $(\alpha=1, \beta)$ (which provides a new model of level 0 players). Right panel of Table 6 shows that each of the two modeling extensions is crucial in improving GCH's overall fit. Below, we dissect the improvement made by both model extensions.

\subsection{Dissecting GCH}

Each of the two modeling innovations can be further dissected to provide the most primitive understanding of what really makes the $\mathrm{GCH}$ model work. Modeling the stereotype bias involves two components: level $k$ 's own stereotype bias and her belief that others have stereotype bias. Similarly, modeling minimum aversion also has two components: aversion in level 0's own behavior and higher levels' response to level 0's aversion. We discuss each of these components in formal terms below and show how each accounts for the improvement of $\mathrm{GCH}$ over $\mathrm{CH}$.

\subsubsection{Allowing for Stereotype Bias: The $\alpha$ Parameter}

When $\alpha=1$, there is no stereotype bias because level $k$ 's beliefs of proportions of lower levels correspond to their true relative proportions. When $\alpha=\infty$, there is maximum stereotype bias because level $k$ believes that opponents are all of same type (and is the most frequently occurring lower level). GCH assumes that level $k$ players exhibit stereotype bias (i.e., $\alpha>1$ ) and they project themselves on others and assume that others (i.e., lower level players) exhibit the same bias too. To separate the effects of level $k$ players' own stereotype bias and their belief that others have stereotype bias, let us denote $\alpha^{*}$ and $\alpha^{* *}$ as parameters for own stereotype bias and belief of others' stereotype bias. Both parameters impact on the expected payoff of level $k$ as follows,

$$
E_{k}\left(\pi_{i}\left(s^{j}\right)\right)=\sum_{j^{\prime}=1}^{m_{-i}} \pi_{i}\left(s^{j}, s^{j^{\prime}}\right)\left\{\sum_{h=0}^{k-1} g_{k}^{*}(h) \cdot P_{h}\left(s^{j^{\prime}} \mid g_{k}^{* *}(h)\right)\right\}
$$

where $g_{k}^{*}(h)$ is the relative proportion with level $k$ 's own stereotype bias $\alpha^{*}$; and the choice

probability of lower levels $P_{h}\left(s^{j^{\prime}} \mid g_{k}^{* *}(h)\right.$ ) (in particular $\left.g_{k}^{* *}(h)\right)$ is based on level $k$ belief of 
lower levels' stereotype bias $\alpha^{* *}$.

In the GCH model, we have $\alpha^{*}=\alpha^{* *}=\alpha$. We set $\alpha^{*}=1$ to suppress level $k$ players' own stereotype bias, but allow them to believe that lower level players have stereotype bias. In

other words, the choice probability $P_{h}\left(s^{j^{\prime}} \mid g_{k}^{* *}(h)\right)$ is conditioned on the belief of $k$ on lower levels having stereotype bias $\alpha^{* *}=\alpha$ and hence $g_{k}^{* *}(h)$. Comparing this to the full GCH model allows us to gauge the effect of own stereotype bias.

Log-likelihood of the best fitted GCH model is -2637.33 with $\tau=1.11, \alpha=2.10$ and $\beta=2.10$, as reported in Table 6 . When we suppress own stereotype bias with $\alpha^{*}=1$ only (and $\left.\alpha^{* *}=\alpha=2.10\right)$, log-likelihood of the model drops to -2675.07. This suggests that own stereotype bias plays a significant role in improving fit. If we were to suppress both stereotype biases with $\alpha^{*}=1$ and $\alpha^{* *}=1$, the further drop in log-likelihood is insignificant at -2675.16. This suggests that level $k$ 's belief of others' stereotype bias does not significantly affect fit. In fact, if one were to suppress only level $k$ 's belief on lower levels' stereotype bias by having $\alpha^{* *}=1$ (and $\alpha^{*}=\alpha$ ), the log-likelihood drops to -2639.56 which is marginally lower than -2637.33 of the full GCH model. Even if we allow the belief that lower levels have extreme stereotype bias $\alpha^{* *}=\infty$, the log-likelihood is -2640.87 . Hence, the model fit is not affected much by big swings in the belief of others' stereotype bias. In summary, we can conclude that modeling level $k$ own stereotype bias matters the most while modeling belief of others' stereotype bias matters the least.

\subsubsection{Allowing for Minimum Aversion: The $\beta$ Parameter}

Right panel of Table 6 shows that allowing for minimum aversion improves the fit significantly. Does $\beta>1$ fit both behaviors of level 0 and higher level players better? Specifically, does minimum aversion provide a better description of level 0 behavior, or does it also describe the belief of higher levels (which determine the behavior of higher levels) better.

To test and quantify the impact of our proposed model of level 0 on the behavior of higher levels, we suppress the model of level 0 in the behavior of higher levels. In other words, we run a model where the belief of higher levels is based on the behavior of a uniformly random level 0 , not the model of level 0 ; but the behavior of level 0 is that prescribed by the model 
of level 0 . Let us denote level 0's minimum aversion by $\beta^{*}$ and higher levels' belief of level 0 aversion by $\beta^{* *}$. In GCH, $\beta^{*}=\beta^{* *}=\beta$. Formally, the modified expected payoff of level 1 and higher from equation (1) is as follows:

$$
E_{k}\left(\pi_{i}\left(s^{j}\right)\right)=\sum_{j^{\prime}=1}^{m_{-i}} \pi_{i}\left(s^{j}, s^{j^{\prime}}\right)\left\{\sum_{h=0}^{k-1} g_{k}(h) \cdot P_{h}\left(s^{j^{\prime}} \mid \beta^{* *}\right)\right\}
$$

while the behavior of level 0 behavior is modeled by $P_{0}\left(s^{j} \mid \beta^{*}\right)$.

The model of higher levels based on the above expected payoff where $\beta^{* *}=1$ (that is, uniform random level 0) together with $\beta^{*}=\beta$ for level 0 behavior yield a log-likelihood of -2691.37. This sizeable drop from -2637.33 suggests that responding to minimum averse level 0 describes the behavior of higher levels more accurately. If we further suppress minimum aversion in level 0 with $\beta^{*}=1$ (in addition to $\beta^{* *}=1$ ), there is a further sizable drop in $\log$-likelihood to -2716.94. Hence, we can conclude that the model of level 0 provides a better description of both level 0 and higher levels.

\section{Applications of GCH Model}

Going beyond calibrating the GCH model on the extensive repertoire of 2-person normal form matrix games, we demonstrate the applicability of the GCH model in two different classes of games with varied game structure. The first application is on the compromise game proposed in Carrillo and Palfrey (2009) where subjects with private signals of their own strengths choose to fight or to compromise. Empirical result shows that the fighting rate is substantially lower than equilibrium prediction of always fight. The second application is on some modified coordination games in Amaldoss, Bettman and Payne (2008) where coordination improves with the addition of an irrelevant alternative. In each application, we take the approach of calibrating on half of the games and validating on another half which has different design parameters.

\subsection{To Fight or To Compromise}

Carrillo and Palfrey (2009) study a game of two-sided private information. In this two-player game, each player is privately assigned a randomly drawn strength $\sigma \in[0,1]$. With his own 
strength, each player decides to either compromise or fight. If at least one player chooses to fight, the player with higher strength is paid 1 and the weaker player is paid 0 . If both choose to compromise, they are both paid $M$. The equilibrium prediction is for both to fight regardless of own strength. Only $61.1 \%$ fighting is observed as opposed to the equilibrium prediction of $100 \%$.

Since a player's decision is irrelevant if the other player decides to fight, we only need to consider the best response function conditioned on the other player choosing compromise. Specifically, the best response function should depend on own strength and will be in the form of cut-point or threshold: suppose own strength is $\sigma$, fight if $\sigma>\sigma^{*}$ for some threshold $\sigma^{*}$, compromise otherwise. The threshold strength is where expected payoff of fighting equals $M$, the payoff for compromise.

To determine whether level $k$ should fight, let us first define $M_{k}(\sigma)$ as the expected payoff of fighting for level $k$ with strength $\sigma$, given that lower levels compromise. In addition, denote $q^{k}(\sigma)$ as the posterior strength distribution of level $k$ given that level $k$ compromises.

Beginning with level 0, the expected payoff of fighting is better than compromising when $\sigma>M$; and the expected payoff of fighting is worse than compromising when $\sigma<M$. Hence with minimum aversion, level 0 will fight with probabilities $P($ fight $\mid \sigma>M)=\frac{\beta}{1+\beta}$ and $P($ fight $\mid \sigma<M)=\frac{1}{1+\beta}$. Conditioned on level 0 compromising, the posterior strength distribution of level 0 is $q^{0}(\sigma)=\frac{\beta}{M \cdot \beta+(1-M)}$ over the range $[0, M]$ and $q^{0}(\sigma)=\frac{1}{M \cdot \beta+(1-M)}$ over the range $[M, 1]$.

The expected payoff of fighting for level 1 with strength $\sigma$ is $M_{1}(\sigma)=\int_{0}^{\sigma} q^{0}\left(\sigma^{\prime}\right) \partial \sigma^{\prime} \cdot 1+$ $\int_{\sigma}^{1} q^{0}\left(\sigma^{\prime}\right) \partial \sigma^{\prime} \cdot 0=\frac{\beta}{M \beta+(1-M)} \cdot \sigma$ for $\sigma<M$. Level 1 is indifferent between compromise and fight at the cutpoint $\sigma^{1}$ where $M_{1}\left(\sigma^{1}\right)=M$. Hence the best response of level 1 is to compromise when $\sigma \leq \sigma^{1}=M \cdot \frac{M \beta+(1-M)}{\beta}$ and fight otherwise. Therefore, the posterior strength distribution of level 1 is $q^{1}(\sigma)=1$ over the range $\left[0, \sigma^{1}\right]$ and $q^{1}(\sigma)=0$ otherwise.

In general, the cumulative strength distribution of lower levels and hence the expected payoff for level $k$ to fight, given all lower levels compromise, can be derived recursively as follows: 


$$
M_{k}(\sigma)=\frac{\sum_{h=0}^{k-1}\left[g_{k}(h) \cdot \int_{0}^{\sigma} q^{h}\left(\sigma^{\prime}\right) \partial \sigma^{\prime}\right]}{\sum_{h=0}^{k-1} g_{k}(h) \cdot \sigma^{h}}
$$

where $g_{k}(h)$ is the level $k$ perceived proportion of level $h, h<k$, as previously defined; and $\sigma^{h}$ is the threshold cutpoint for level $h$.

The cut-point of level $k$ is derived from $M_{k}\left(\sigma^{k}\right)=M$. The best response of level $k$ is therefore compromise if $\sigma \leq \sigma^{k}$, otherwise fight. Note that $\sigma^{k} \leq \sigma^{k-1}$. As thinking level $k$ increases, the range of compromise $\left[0, \sigma^{k}\right]$ shrinks and the chance of compromise gets smaller. In the limit, we will observe the best response of always fight, which is the equilibrium prediction.

Two game sessions in Carrillo and Palfrey (2009) were run in simultaneous move; one with $M=\frac{7}{18}=0.39$ and the other with $M=0.50$. We calibrate on the session with $M=0.50$ and validate on the session with $M=0.39 .^{8}$ The estimation result and the parameter estimates are reported in top panel of Table 7.

GCH offers the best fit and LK the worse. The better fit of GCH over CH and LK in calibration is statistically significant at $1 \%$. The performance of $\mathrm{GCH}$ in validation is also substantially better than the $\mathrm{CH}$ and the LK models. The $\tau$ estimates from all three models are fairly close to one another staying in the range of [1.24,1.60]. The stereotype bias parameter $\alpha$ is equal to 2 , which is close to the value of 2.1 from the matrix games. The minimum aversion parameter $\beta$ is however lower (than that of the matrix game) at 1.41, indicating that level 0 favors the never-worst strategies $41 \%$ more. The following two figures would better illustrate where the GCH model improves over the LK model (and the CH model).

One can observe from both figures that the GCH model (represented by the bold solid step line) fits the data better than the LK model (represented by the dotted line) in two ways. First, the GCH model predicts a wider range of fight rate over the entire strength distribution. This effect is generated by the model of level 0. Second, the GCH model steps up the fight rate at the right strength level compared to the LK model; in other words, the

\footnotetext{
${ }^{8}$ Calibrating on the $M=0.39$ session yields a substantially better fit for $\mathrm{GCH}$ when compared to $\mathrm{CH}$ and LK where log-likelihood for GCH is -141 versus -181 for CH and -209 for LK. One reason that GCH fits better for $M=0.39$ is due mainly to the higher contribution from capturing minimum aversion.
} 
Table 7: Model Fits and Estimates for the 2 Applications

\begin{tabular}{|c|c|c|c|c|c|c|c|}
\hline Model & & $\mathrm{GCH}$ & & $\mathrm{CH}$ & & LK & \\
\hline \multicolumn{8}{|l|}{ Carrilo and Palfrey (2009) $)^{\frac{1}{}}$} \\
\hline \multicolumn{8}{|l|}{ Log-likelihood } \\
\hline Calibration (560 observations) & & -215 & & -217 & & -239 & \\
\hline$\%$ Improvement of GCH over the model & & & & $1.0 \%$ & & $11.1 \%$ & \\
\hline Validation (560 observations) & & -172 & & -181 & & -209 & \\
\hline$\%$ Improvement of GCH over the model & & & & $5.1 \%$ & & $21.2 \%$ & \\
\hline Bayesian Information Criterion (BIC) & & 449 & & 440 & & 484 & \\
\hline LL Ratio with GCH & & & & 4 & & 48 & $*$ \\
\hline \multirow[t]{3}{*}{ Parameter Estimates } & $\tau$ & 1.42 & $*$ & 1.60 & $*$ & 1.24 & $*$ \\
\hline & $\alpha$ & 2.00 & $*$ & - & & - & \\
\hline & $\beta$ & 1.41 & $*$ & - & & - & \\
\hline \multicolumn{8}{|l|}{ Amaldoss, Bettman and Payne (2008) $)^{2}$} \\
\hline \multicolumn{8}{|l|}{ Log-likelihood } \\
\hline Calibration (720 observations) & & -1051 & & -1074 & & -1109 & \\
\hline$\%$ Improvement of GCH over the model & & & & $2.2 \%$ & & $5.5 \%$ & \\
\hline Validation (720 observations) & & -1130 & & -1137 & & -1168 & \\
\hline$\%$ Improvement of $\mathrm{GCH}$ over the model & & & & $0.6 \%$ & & $3.3 \%$ & \\
\hline Bayesian Information Criterion (BIC) & & 2122 & & 2155 & & 2225 & \\
\hline LL Ratio with GCH & & & & 46 & $*$ & 116 & $*$ \\
\hline \multirow[t]{3}{*}{ Parameter Estimates } & $\tau$ & 1.78 & $*$ & 2.48 & $*$ & 2.04 & $*$ \\
\hline & $\alpha$ & 3.49 & $*$ & - & & - & \\
\hline & $\beta$ & 1.49 & $*$ & - & & - & \\
\hline \multicolumn{8}{|c|}{${ }^{1}$ Models calibrated on dataset with $\mathrm{M}=0.50$ (321 fight) and validated on $\mathrm{M}=0.39$ (368 fight) } \\
\hline \multicolumn{8}{|c|}{${ }^{2}$ Models calibrated on set 6 and validated on set 5 , each with 2 sessions AA'B and ABB'. } \\
\hline${ }^{3}$ Bold indicates best performing model. & & & & & & & \\
\hline
\end{tabular}


FIGURE 2

Fight Rate over Strength $[0,1]$ with $M=\frac{7}{18}$

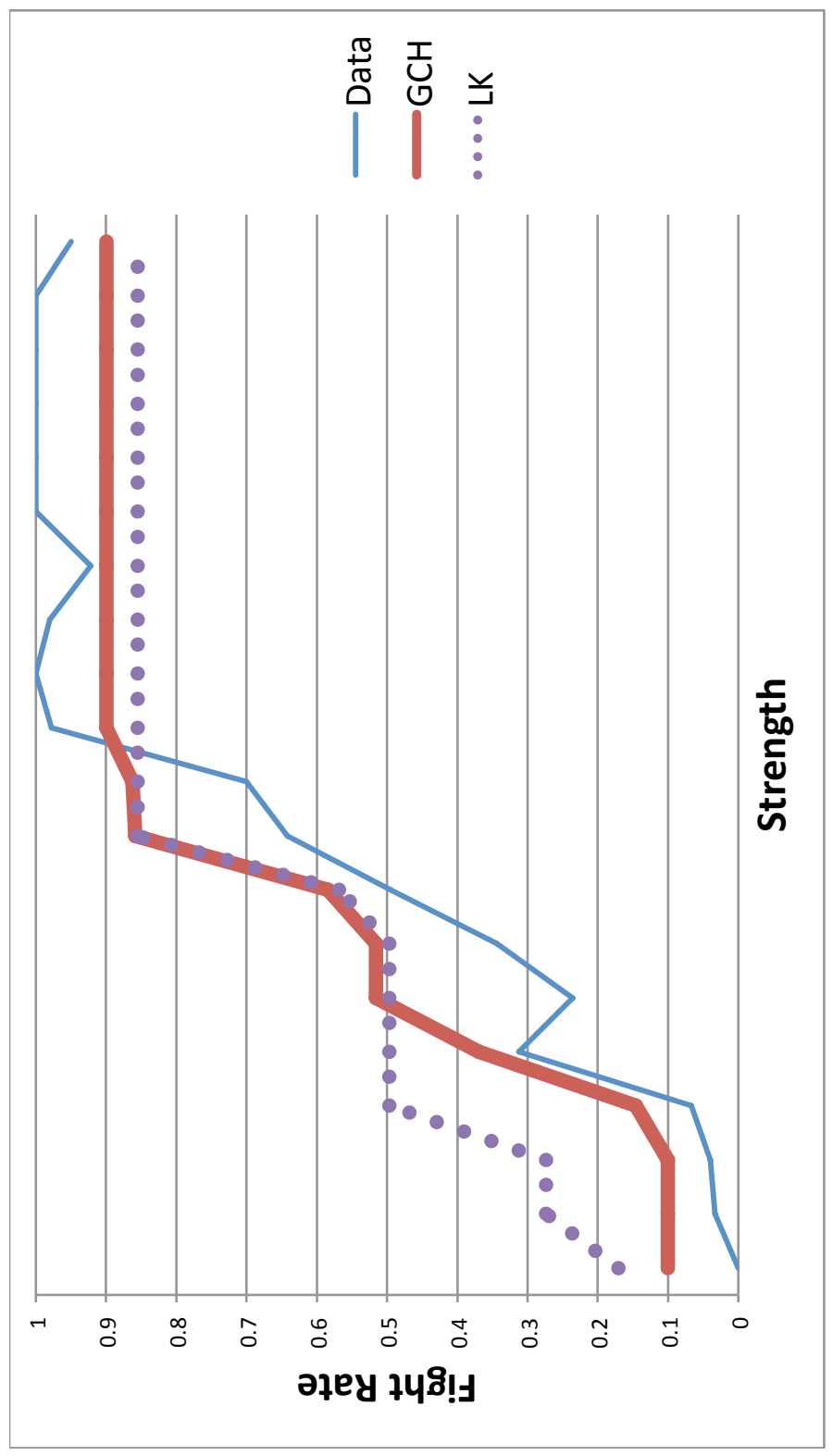


FIGURE 3

Fight Rate over Strength $[0,1]$ with $M=0.50$

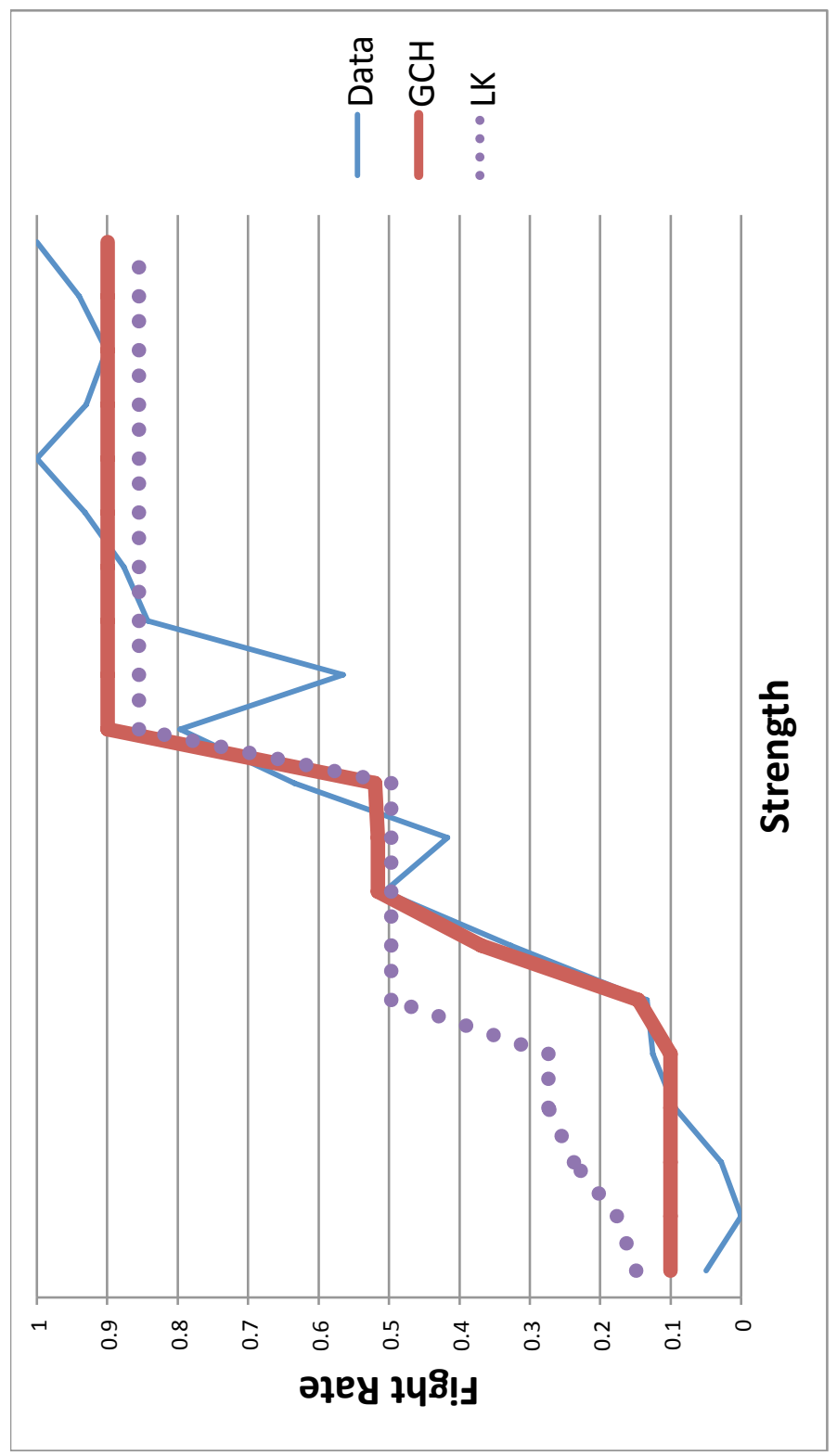


predicted fight rate of LK is almost always higher than the GCH model except in the higher end of strength. This effect is generated by the model of opponents.

\subsection{Coordination by Irrelevant Alternative}

Amaldoss, Bettman and Payne (2008) demonstrated how coordination could be improved by adding a weakly dominated alternative. In a 2x2 nonzero sum game, row player chooses between A and B and column player chooses between L and R. There are two pure strategy coordinating equilibria which pay better, $(\mathrm{A}, \mathrm{R})$ and $(\mathrm{B}, \mathrm{L})$. When a weakly dominated (by one of the two original strategies) alternative was added to the choice set of the row player, Amaldoss et al (2008) showed that the choice proportion of the dominating strategy increased. Specifically, when A' (weakly dominated by A) was added, the choice proportion of A increased from $47.22 \%$ to $59.45 \%$. Similarly, when B' is added, the proportion of B increased from $52.78 \%$ to $83.12 \%$ (see Table 5 of Amaldoss et al (2008)). Column players chose $\mathrm{R}$ more frequently when A' was added, increased from $51.81 \%$ to $72.92 \%$. Hence, both row and column players had higher proportion of coordination at (A, R), increased from an average of $22.22 \%$ to $41.67 \%$. Column players also chose L more frequently when B' was added, increased from $48.19 \%$ to $75.14 \%$. This resulted in better coordination at (B, L), increased from an average of $23.20 \%$ to $63.20 \%$ (see Table 7 of Amaldoss et al (2008)).

Similar to Amaldoss et al (2008), we analyze strategic thinking using the data generated in their study 2, datasets 5 and 6 . The two datasets use different payoff matrices. Each dataset has two sessions, one with row strategies $\left(\mathrm{A}, \mathrm{B}, \mathrm{A}^{\prime}\right)$ and another with $\left(\mathrm{A}, \mathrm{B}, \mathrm{B}^{\prime}\right) .{ }^{9}$ Column players in both sessions have column strategies (L, R). We calibrate on dataset 6 and validate on dataset $5 .{ }^{10}$ Bottom panel of Table 7 reports the parameters, the calibration and

\footnotetext{
${ }^{9}$ Minimum aversion picks up the asymmetric dominance in the $\left(\mathrm{A}, \mathrm{B}, \mathrm{A}^{\prime}\right)$ sessions where $\mathrm{A}$ is favored by level 0 row player but in the (A,B,B') session, $B$ is not favored by level 0 row player. In the (A,B,B') session the weakly dominant alternative B pays the minimum (same as B') when column player picks $\mathrm{R}$, hence no strategy is favored by level 0 row player. In short, $(\mathrm{A}, \mathrm{B}, \mathrm{B}$ ') is a game with a specific form of asymmetric dominance which GCH model does not cover.

${ }^{10}$ Calibrating on dataset 5 yields a substantially better fit for GCH when compared to $\mathrm{CH}$ and LK where log-likelihood for GCH is -1026 versus -1111 for $\mathrm{CH}$ and -1160 for LK. One reason that GCH fits better in dataset 5 is due to the even higher contribution from capturing minimum aversion.
} 
validation results.

GCH provides an improvement in log-likelihood of 23 points and 58 points over CH and LK respectively. GCH also has a significant lead over the $\mathrm{CH}$ and the $\mathrm{LK}$ model in validation. The parameter estimates of the GCH model are $\tau=1.78, \alpha=3.49$ and $\beta=1.49$, compared to $\tau=2.48$ for the $\mathrm{CH}$ model and $\tau=2.04$ for the LK model. The average thinking step is about 2 when we look at the $\tau$ estimates from the 3 models. Stereotype bias seems to be fairly strong at 3.49. When coupled with the $\tau$ estimates, it suggests that level 2 and above respond mostly to level 1 . Never worst strategies are $49 \%$ more favored by level 0 .

To further understand the differences among the three models, we describes the predicted behaviors of level $k$ players using the $\left(\mathrm{A}, \mathrm{B}, \mathrm{A}^{\prime}\right.$ ) session in dataset 6 (payoff matrix reproduced in Table 8).

Table 8: (A,B,A') session in Dataset 6 from Amaldoss et al [2008]

\begin{tabular}{l|lc}
\hline \hline & L & $\mathrm{R}$ \\
\hline A & $\mathbf{1 6 , 1 6}$ & 20,24 \\
A' & $\mathbf{8}, 22$ & $20, \mathbf{1 2}$ \\
B & 24,20 & $\mathbf{8 , 8}$ \\
\hline \hline \multicolumn{3}{l}{ Note: Payoffs in bold indicate the minimum payoff for } \\
the corresponding columns or rows. The never worst \\
set for the row player consists of strategy A which \\
weakly dominates strategy A'. The never worst set \\
for column player is empty. There are two equilibria, \\
namely (A,R) and (B,L).
\end{tabular}

As indicated in Table 8, strategy A in the Never Worst set is preferred by level 0 row player while level 0 column player randomizes between $\mathrm{L}$ and $\mathrm{R}$. $\mathrm{GCH}$ predicts that the behavior of row player level 3 and above stabilizes onto strategy A and the column player level 2 and above stabilizes onto strategy R. Hence, GCH predicts the game to stabilizes onto (A, R), one of the two equilibria, for players level 3 and above. While the $\mathrm{CH}$ model predicts that row player level 5 and above chooses A and column player level 5 and above chooses R. In other words, the $\mathrm{GCH}$ and $\mathrm{CH}$ models predict higher level players converging onto one of 
the two equilibria. More importantly, the equilibrium converged on is the one coordinated by the irrelevant alternative A'. Whereas, the LK model predicts that behaviors of both row and column oscillate among the available strategies infinitely.

While the aggregate prediction of the $\mathrm{GCH}$ and $\mathrm{CH}$ models are similar, the rationale behind is different. With a higher $\tau=2.48$ compared to GCH's 1.78, the $\mathrm{CH}$ model suggests that most people think a lot harder to coordinate and only thinking beyond level 4 gets to coordination. While GCH suggests that most people have stereotype bias and best respond largely to the most common occurring type. Thinking beyond level 2 is sufficient to get to coordination. Most other datasets seem to corroborate more with the latter rationale than the former.

\section{Conclusion}

This paper presents a general model of cognitive hierarchy which incorporates a more general model of level 0 and a more general model of opponents. The general model of level 0 posits that level 0 is averse to strategies that yield minimum payoff in at least one scenario. Our empirical result suggests that the "never-worst" strategies are more than twice preferred over these "minimum" strategies. we call this effect the minimum aversion effect. It incorporates the asymmetric dominance effect, the compromise effect and captures preference over dominant strategies. This general model of level 0 is not only parsimonious, it is also effective at explaining behavior with significant improvement in fit. It not only explains level 0 better, it is also explaining higher level behaviors better.

The general model of opponent captures the whole spectrum of stereotype bias. It begins with the basic $\mathrm{CH}$ model with no stereotype bias to the Level $\mathrm{M}$ model with maximum stereotype bias, a close cousin of Level K. When a player has maximum stereotype bias, he only focuses on responding to the behavior of the most frequently occurring type known to him, i.e. among those with lower thinking levels. Our empirical result suggests that there is a mild level of stereotype bias. Incorporating stereotype bias in the model improves the fit significantly. This improvement in fit comes from two sources: the accurate belief of the stereotype bias in the lower types and the stereotype bias in one's own action. 
With careful calibration and validation using the rich repertoire of datasets, the GCH model provides a very robust and decent performance in one-shot games. More importantly, it provides an intuitive and viable explanation of the observed behaviors. We believe the model has wider applicability. In the last section, we offers two application examples of this Generalized $\mathrm{CH}$ model.

For future research direction, we suggest that the GCH model can be applied with some perturbations to other economic applications to provide a better understanding of the underlying behaviors. In particular, our model of opponent can be parameterized to explain how stereotype bias might vary depending on how subjects' prior is primed; our model of level 0 can be parameterized to capture the potential impact of game structure, and more specifically the impact of payoff structure.

\section{Appendix 1: Entry Game Analysis for the LM model}

We have $e(0, d)=\frac{1}{2}$ for all $d$, and

$$
E_{k}(k-1, d)=\left\{\begin{array}{lll}
e(k-1, d) & \text { if } \quad k \leq \text { mode } \\
e(\text { mode }, d) & \text { if } \quad k>\text { mode }
\end{array}\right.
$$

For $k \geq 1$

$$
e(k, d)=\left\{\begin{array}{lll}
0 & \text { if } & E_{k}(k-1, d)>d \\
1 & \text { if } & E_{k}(k-1, d)<d
\end{array}\right.
$$

We shall derive the condition on $\tau$ for the Poisson distribution when total entries is monotonically increasing in demand. Given that the entry function of level $k$ only depends on a single lower level (whether it is $k-1$ or mode of the distribution), there is only one single cutpoint as $d$ increases. For $d<\frac{1}{2}$, level 1 will stays out, level 2 enters, and continuing with odd 
level stay out and even levels enter, until the mode. If mode is even, then all levels above mode stay out, otherwise, they enter. Hence, total entry is $(1 / 2) f(0)+f(2)+f(4)+\cdots+f($ mode $)$, if mode is even; otherwise it is $(1 / 2) f(0)+f(2)+f(4)+\cdots+f$ (mode -1$)+f$ (mode + $1)+f($ mode +2$)+\cdots$. For $d>\frac{1}{2}$, level 1 enters since half of level 0 enter. Level 2 stays out, and continuing with odd levels enter and even levels stay out until the mode. If mode is even, then all levels above mode enter, otherwise, they stay out. Hence, total entry is $(1 / 2) f(0)+f(1)+f(3)+\cdots+f($ mode -1$)+f($ mode +1$)+f($ mode +2$)+\cdots$, if mode is even; otherwise it is $(1 / 2) f(0)+f(1)+f(3)+\cdots+f($ mode $)$.

If mode is even, the condition of monotonically increasing entries requires total entries in $d<1 / 2$ to be less than total entries in $d>1 / 2$. In other words, $1-f(0)>$ $2(f(2)+f(4)+\cdots+f($ mode $))$, this is always true for the unimodal Poisson distribution given the $f(0)$ is exponentially decreasing in $\tau$. If the mode is odd, we have the condition that $1-f(0)<2(f(1)+f(3)+\cdots+f($ mode $))$, this condition is satisfied when $\tau \leq 1.256$. Hence, total entries is monotonically increasing in demand when $\tau \leq 1.256$ in a Poisson distribution of thinking levels.

\section{References}

Amaldoss, Wilfred, James R. Bettman and John W. Payne "Biased but Efficient: An Investigation of Coordination Facilitated by Asymmetric Dominance," Marketing Science, 27(5), (2008), 903-921.

Binmore, Kenneth, Joe Swierzbinski and Chris Proulx, "Does Maximin Work? An Experimental Study," Economic Journal, 111, (2001), 445-464.

Bloomfield, Robert, "Learning a Mixed Strategy Equilibrium in the Laboratory," Journal of Economic Behavior and Organization, 25, (1994), 411-436.

Camerer, Colin F., Teck-Hua Ho and Juin-Kuan Chong, "A Cognitive Hierarchy Theory of One-shot Games," Quarterly Journal of Economics, 119(3), (2004), 861-898. 
Carrillo, Juan D. and Thomas R. Palfrey, "The Compromise Game: Two-Sided Adverse Selection in the Laboratory," American Economic Journal: Microeconomics, 1(1), (2009), 151-181.

Cooper, David and John Van Huyck, "Evidence on the Equivalence of the Strategic and Extensive Form Representation of Games," Journal of Economic Theory, 110(2), (2003), 290-308.

Costa-Gomes, Miguel and Vincent Crawford, "Cognition and Behavior in TwoPerson Guessing Games: An Experimental Study," American Economic Review, 96, (2006), 1737-1768.

Costa-Gomes, Miguel, Vincent Crawford and Bruno Broseta, "Cognition and Behavior in Normal-form Games: An Experimental Study," Econometrica, 69(5), (2001), 1193-1235.

Crawford, Vincent, "Boundedly Rational versus Optimization-Based Models of Strategic Thinking and Learing in Games", Journal of Economic Literature, 51, (2013), $512-527$.

Crawford, Vincent and Nagore Iriberri, "Fatal Attraction: Salience, Naivete, and Sophistication in Experimental Hide-and-Seek Games," American Economic Review, 97(5), (2007), 1731-1750.

Goeree, Jacob, Charles Holt and Thomas Palfrey, "Risk Averse Behavior in Generalized Matching Pennies Games," Games and Economic Behavior, 45(1), (2003), 97-113.

Lieberman, Bernhardt, "Experimental studies of conflict in some two-person and three-person games," In J. H. Criswell, H. Solomon, and P. Suppes (Eds.), Mathematical Models in Small Group Processes. Stanford: Stanford University Press, (1962), 203-220.

Messick, David M., "Interdependent Decision Strategies in Zero-sum Games: A 
Computer-controlled Study," Behavioral Science, 12, (1967), 33-48.

Mookerjee, Dilip and Barry Sopher, "Learning and Decision Costs in Experimental Constant-sum Games," Games and Economic Behavior, 19, (1997), 97-132.

Nagel, Rosemarie, "Unraveling in Guessing Games: An Experimental Study," American Economic Review, 85(5), (1995), 1313-1326.

Ochs, Jack, "Games with Unique, Mixed Strategy Equilibria: An Experimental Study," Games and Economic Behavior, 10, (1995), 202-217.

O'Neill, Barry, "Nonmetric Test of the Minimax Theory of Two-person Zerosum Games," Proceedings of the National Academy of Sciences, 84, (1987), 2106-2109.

Rapoport, Amnon and Amaldoss, Wilfred, "Mixed Strategies and Iterative Elimination of Strongly Dominated Strategies: An Experimental Investigation of States of Knowledge," Journal of Economic Behavior and Organization, 42, (2000), 483-521.

Rapoport, Amnon and Boebel, Richard B., "Mixed Strategies in Strictly Competitive Games: A Further Test of the Minimax Hypothesis," Games and Economic Behavior, 4, (1992), 261-283.

Stahl, Dale O., and Paul Wilson, "Experimental Evidence on Players' Models of Other Players," Journal of Economic Behavior and Organization, 25, (1994), 309-327.

Stahl, Dale O., and Paul Wilson, "On Players Models of Other Players: Theory and Experimental Evidence," Games and Economic Behavior, 10, (1995), 218-254.

Tang, Fang-Fang, "Anticipatory Learning in Two-person Games: Some Experimental Results," Journal of Economic Behavior and Organization, 44, (2001), 221-32. 